Article

\title{
Design of a Dual-Technology Fusion Sensor Chip with a Ring Electrode for Biosensing Application
}

\author{
Cheng Ma ${ }^{1}$, Jin Zhu ${ }^{1,2, *} \mathbb{C}$, Xiaolong $\mathrm{Li}^{1,2}$ and Wei Zheng ${ }^{1,2}$ \\ 1 College of Electronic and Information, Jiangsu University of Science and Technology, \\ Zhenjiang 212003, China; 162210304221@stu.just.edu.cn (C.M.); lixiaolong@just.edu.cn (X.L.); \\ zhengwei@just.edu.cn (W.Z.) \\ 2 Zhenjiang Laboratory of Information Sensing and Transmission Technology for Smart Ocean, \\ Zhenjiang 212000, China \\ * Correspondence: oscar@just.edu.cn; Tel.: +86-511-84401153
}

Received: 29 January 2019; Accepted: 18 February 2019; Published: 23 February 2019

\begin{abstract}
Quartz crystal microbalance (QCM) is still a new high-precision surface detection technique. However, the adsorption quality detected by the QCM currently contains a solvent-coupling quality and cannot separate the actual biomolecular mass. Local surface plasmon resonance (LSPR) can detect the mass of biomolecules, but requires a certain contrast between the solvent of the surrounding medium and the refractive index of the adsorbed layer. The sensor chip, combining two compatible technologies, can realize the simultaneous detection of biomolecules and improve the refractive index sensitivity. The structure of our chip is to prepare the ring-shaped gold electrode on the upper surface of the quartz crystal, the circular gold electrode on the bottom surface, and the spherical gold nanoparticles arrays in the center region of the ring electrode to form a QCM/LSPR dual-technology chip. Through simulation, we finally get the size of the best energy trap by the two electrodes on the upper surface and the lower surface: the ring-top electrode with a thickness of $100 \mathrm{~nm}$, an inner diameter of $4 \mathrm{~mm}$, and an outer diameter of $8 \mathrm{~mm}$; and the bottom electrode with a thickness of $100 \mathrm{~nm}$ and a radius of $6 \mathrm{~mm}$. By comparing the refractive index sensitivity, we chose a spherical gold nanoparticle with a radius of $30 \mathrm{~nm}$ and a refractive sensitivity of $61.34 \mathrm{~nm} / \mathrm{RIU}$ to design the LSPR sensor chip.
\end{abstract}

Keywords: gold nanoparticles; quartz crystal microbalance (QCM); local surface plasmon resonance (LSPR); gold electrode; dual-technology chip

\section{Introduction}

With the development of science and technology, the research of surface probe technology is deepening. The use of single technology is not enough to obtain the parameters of the complex biofilm structure. Therefore, combining the two technologies can expand the amount of information detected by the system and improve the reliability of the detection information and precision. Quartz crystals have high sensitivity, small size and strong vibration resistance, so they can be used to measure the parameters, which other instruments with low precision cannot measure [1]. The principle of quartz crystal microbalance (QCM) is to use the piezoelectric effect [2-4] of quartz crystal to convert the subtle mass change on the quartz crystal into a change in the vibration frequency. The detection accuracy of QCM is proportional to the resonant frequency. QCM was originally applied to air or a vacuum to detect substances adsorbed on its surface. With the development of science and technology, QCM has progressed to have more applications in liquid [5-9]. However, QCM is only suitable for rigid films that are thin enough and evenly spread relative to quartz crystals. Quartz crystal microbalance with dissipation monitoring (QCM-D) can determine whether the surface adsorbs soft/viscoelastic 
materials or rigid material by observing the change of dissipation value, $\Delta D$, and can also monitor the viscoelasticity and structural changes of the material in real time. However, because the quality of QCM-D detection included the coupling quality of the solvent, the quality of the actual biomolecule "dry mass" cannot be measured [10]. Local surface plasmon resonance (LSPR) is an optical detection technology with high sensitivity and real-time detection of binding between biomolecules. When light waves having the same electron frequency as the metal nanostructures are irradiated onto the metal surface, the charge on the metal surface oscillates, the electromagnetic field is enhanced, and the refractive index change in the vicinity of the metal nanostructure becomes sensitive, so that the sensitivity is improved [11]. In liquid, LSPR measures the "dry mass" of the biomolecules but requires different refractive index between the adsorbed layer and the solvent. The structural properties of the adsorbed layer cannot be obtained by LSPR alone. With the rapid development of QCM's sensing technology, QCM-based sensors have been widely used in applications such as the detection of chemicals, biomolecules, and bacteria. Applying different materials to the surface of QCM allows it to be used for non-enzymatic glucose detection [12], determination of protein concentration [13], determination of prostate-specific antigen (PSA), and detection of PSA- $\alpha 1$-antichymotrypsin (ACT) complex (75\%) in human serum [14]. The QCM immunosensor can also be used to detect epidermal growth factor receptor (EGFR) $[15,16]$ and the like. When LSPR is excited, both absorption and scattering are greatly enhanced. Therefore, spectroscopy is the simplest method of detecting LSPR on metal nanostructures and is typically based on extinction, or scatterometry, measurements. Extinction is commonly used to characterize systems containing large amounts of nanostructures, such as nanoparticle colloids. The extremely intense and highly restricted electromagnetic field caused by LSPR provides very sensitive probes to detect small changes in the dielectric environment around the nanostructures, which is particularly attractive for sensing applications $[17,18]$.

If we run both technologies on the same chip at the same time, we can combine the advantages of both technologies. By complementing the advantages of the two technologies, it is possible to obtain data that cannot be obtained by a single measurement technique. QCM-D can measure the total mass of the adsorbed molecular film (including the coupling solvent mass). LSPR can measure the actual adsorbed molecular mass, and we can get the coupling solvent quality by subtracting the molecular mass from the total mass. Both the gold electrode on top of the traditional QCM-D sensor and the gold nanodisc have a layer of silicon dioxide spacer. Using the principle of light reflection, the $\mathrm{SiO}_{2}$ spacer layer through which the light passes is then reflected by the gold electrode at the top, so that the light is twice irradiated to the gold nanodisc. Traditional QCM-D sensors are currently produced and commercialized. Hao et al. [19] have proposed to fabricate a $400 \mu \mathrm{m}$ diameter window in the center of a circular gold electrode under a conventional QCM device, and to prepare a $400 \mu \mathrm{m} \times 400 \mu \mathrm{m}$ array of $\mathrm{Au}$ LSPR nanodisc in the center of the upper circular gold electrode. Using the light transmission principle to illuminate the Au LSPR nanodisc, Ferhan et al. [20] have combined traditional QCM-D with local surface plasmon (LSPR) and studied a combined measurement method. For better research, they have integrated the reflective mode LSPR sensor with the traditional QCM-D instrument platform and used a titanium oxide-coated sensing substrate to study vesicle adsorption and amphipathicity.

The structure of the chip we designed was compared with the conventional QCM-D device, and the top circular gold electrode was replaced with a ring-shaped gold electrode, and the optimal ring gold electrode size was obtained by ANSYS software simulation. Using a pipette to take a small amount of nanosolution titrated in the center of the ring-shaped gold electrode is equivalent to directly preparing on the quartz crystal, allowing it to dry naturally at room temperature. Finally, gold nanoparticles with narrow distribution are adsorbed on the surface of the quartz crystal. The preparation of spherical nanoparticles by a conventional method is simple, and they were directly placed on a quartz wafer without large influence on the electrode structure and the conductivity of the electrodes. The top gold electrode is ring-shaped, and the fundamental frequency of the gold electrode quartz crystal changes by $\Delta f=8574.3 \mathrm{~Hz}$, which is completely negligible compared with the chip's fundamental frequency $f_{s}=4.98 \mathrm{MHz}$. In terms of LSPR, our LSPR spectrum measurement 
works in reflection mode. The light is vertically irradiated onto the light-sensitive area composed of the gold nanospheres, and the light is irradiated onto the gold electrode through the quartz crystal and is vertically reflected back, so that the light is twice irradiated to the gold nanodisc array. The nanoparticle undergoes two LSPR spectral absorptions by one reflection and two transmissions, ultimately enhancing the spectral intensity.

\section{Materials and Methods}

\subsection{Theory}

QCM is a kind of high-precision measuring instrument. The quartz crystal sensor uses AT-cut oscillator [21] with metal electrode prepared on both sides of the quartz crystal. After the electrodes are connected to the wires, the chips are encapsulated to form the quartz crystal resonator. As early as 1959, German physicist Gunter Sauerbrey discovered and pointed out that the decline of the crystal's resonant frequency was proportional to the mass of the surface attachment [22]. However, it only worked in air or vacuum environments, and in liquids, the Sauerbrey equation cannot be established because the viscosity of the liquid dissipates energy. In 1996, Rodahl [23] proposed equations for the change of liquid phase dissipative factor $(\Delta D)$ :

$$
\Delta D=2\left(\frac{f_{0}}{n}\right)^{1 / 2}\left(\frac{\varepsilon Q_{1}}{\pi \mu Q_{q}}\right)^{1 / 2} .
$$

Unlike QCM, QCM-D could measure both the change of quartz crystal frequency and the change of the dissipation value. QCM-D obtains frequency change $(\Delta f)$ by monitoring the change in molecular mass adsorbed on the sensor, such as the Sauerbrey formula:

$$
\Delta f=-2.26 \times 10^{-6} f^{2} \Delta m / A,
$$

and the dissipative value change $\Delta D$ is obtained by the viscoelasticity of the adsorption mass, according to the attenuation formula

$$
\begin{gathered}
A(t)=A_{0} \cdot \exp (-t / \tau) \cdot \sin (2 \pi f t+\varphi), \\
D=1 / \pi f t .
\end{gathered}
$$

Compared with SPR sensor technology, the LSPR technique uses noble metal nanoparticles with special optical properties as the carrier of the sensitive membrane while retaining SPR technology, which makes the sensor device simple and more sensitive to the medium environment around the nanoparticles [24,25]. LSPR is an optical phenomenon. When the incident light is irradiated on the surface of precious metal particles, the coupling of the noble metal nanoparticles produces a plasma resonance that produces one or more maximum values on the extinction efficiency distribution curve $[26,27]$. The extinction efficiency is related to the size and shape of the nanoparticles [28,29].

We designed a photoelectric sensor, which combines QCM-D and LSPR technology to achieve complementary advantages. QCM-D provides a lot of molecular and coupling solvents for the sensor. The environment meets the requirement for LSPR to measure the mass of biomolecules in liquid and make up for the shortcomings of QCM-D detection quality, including the coupling quality of the solvent.

\subsection{Structure}

The QCM/LSPR dual-technology chip integrates reflectance mode LSPR into a QCM, which has a ring-shaped gold electrode. The ring-shaped gold electrode is prepared as the top electrode, and a circular gold electrode is prepared as the bottom electrode. An alternating current (AC) voltage of $5 \mathrm{~V}$ is applied on the upper and lower electrodes to resonate the crystal. The chip structure is shown in Figure 1. The paper obtains the best electrode size by finite element analysis [30]. Gold nanoparticles were synthesized by reduction of $\mathrm{HAuCl}_{4}$ solution with sodium citrate solution. Nanoparticles were 
immobilized by drop-evaporation from the aqueous synthesized solution. The droplet was placed on the quartz in the inner region of the ring electrode and dried at ambient conditions. This approach allowed for the investigation of the LSPR spectrum, which was scattered by nanoparticles. In order to improve the uniformity of the nanoparticle size and the reproducibility of the experiment, a second method can be employed in later experiments to combine the gold nanoparticles with QCM. First, $40 \mathrm{~mL}$ of $1.40 \mathrm{mM} \mathrm{HAuCl}_{4}(\mathrm{mM}=\mathrm{mmol} / \mathrm{L})$ and $400 \mu \mathrm{L}$ of $0.213 \mathrm{M}$ cysteamine hydrochloride were mixed in a glass vial with a capacity of $100 \mathrm{~mL}$. The mixture was then stirred at room temperature and under dark conditions for $20 \mathrm{~min}$. After $20 \mathrm{~min}, 10 \mathrm{~mL}$ of freshly prepared $10 \mathrm{mM} \mathrm{NaBH}$ was quickly added to the above mixture, and then regenerated and stirred for $30 \mathrm{~min}$ to obtain positively charged gold nanoparticles stabilized by cysteamine molecules [31]. Niidome et al. obtained gold nanoparticles AuNPsCH(+) stabilized by cytoamine [32]. After obtaining $\mathrm{AuNPsCH}(+)$, the suspension with a concentration equal to $5 \mathrm{mg} \cdot \mathrm{L}^{-1}$ was sent to the surface of the QCM chip at a steady flow rate of $0.08 \mathrm{~mL} \cdot \mathrm{min}^{-1}\left(1.33 \times 10^{-3} \mathrm{~cm}^{3} \cdot \mathrm{s}^{-1}\right)$ by a microfluidic pump. The AuNPsCH(+) in the solution was deposited for 10 to $300 \mathrm{~min}$ [33].

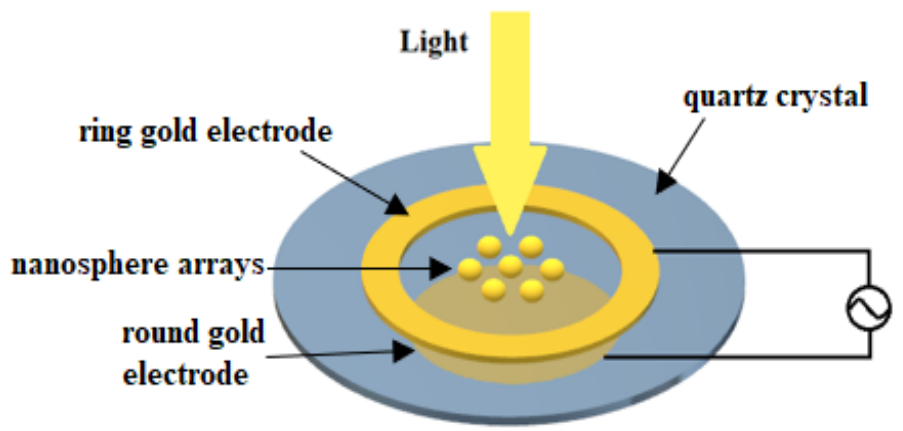

Figure 1. Structure of quartz crystal microbalance (QCM) / local surface plasmon resonance (LSPR) chip.

Since the gold nanoparticles have stable chemical properties and high optical sensitivity, the gold nanoparticle arrays were placed in the central region of the ring-shaped gold electrode [34-37]. When the beam is directed at the center of the ring electrode, the nanoparticles absorb the beam for the first time. Then, the light beam is transmitted through the quartz crystal and irradiated to the gold electrode at the bottom. The light beam is irradiated onto the gold nanoparticle by the reflection of the bottom gold electrode so that the nanoparticle absorbs the light beam for the second time, thereby enhancing the absorption spectral intensity and improving the optical sensitivity. The shape and size of nanoparticles in gold nanoarrays affect the absorption peak of the spectrum. In this paper, the spherical gold nanoparticles are selected to design the LSPR sensor chip.

\section{Procedures}

\subsection{Electrode Size}

We used the finite element analysis method to find the electrode size of the best energy-trapping graph by ANSYS software. QCM consists of a ring-shaped gold electrode above a quartz crystal and a circular gold electrode at the bottom. The inner and outer radius and thickness of the ring-shaped gold electrode, as well as the radius and thickness of the circular gold electrode, have a direct impact on the performance of QCM. We chose the electrodes with a thickness of $100 \mathrm{~nm}$ and a quartz crystal with a thickness of $333 \mu \mathrm{m}$ to study the effect of the size of the electrode radius on the resonant frequency of QCM [38].

For the top gold electrode, we first select the ring-shaped electrode with an inner radius of $4 \mathrm{~mm}$ and an outer radius of $6 \mathrm{~mm}$ to obtain the best energy-trapping diagram and corresponding vibration displacement curve. Figure 2 is an energy-trapping diagram of two subsets extracted from 
the simulation results of vibration mode. Figure 3 shows their vibrational displacement curves along the corresponding radius.

$$
\begin{aligned}
& \text { NODAL SOLUTION } \\
& \begin{array}{l}
\text { STEP }=1 \\
\text { SUB }=43
\end{array} \\
& \begin{array}{l}
\text { SUB }=43 \\
\text { FREQ }=.501 \mathrm{E}+07
\end{array} \\
& \text { UX } \quad \text { (AVG) } \\
& \begin{array}{l}
\text { RSYS }=0 \\
\text { DMX }=167.685
\end{array} \\
& \begin{array}{l}
\text { DMX }=167.685 \\
\text { SMN }=-167.685
\end{array} \\
& \begin{array}{l}
\text { SMN }=-167.685 \\
\text { SMX }=167.685
\end{array}
\end{aligned}
$$
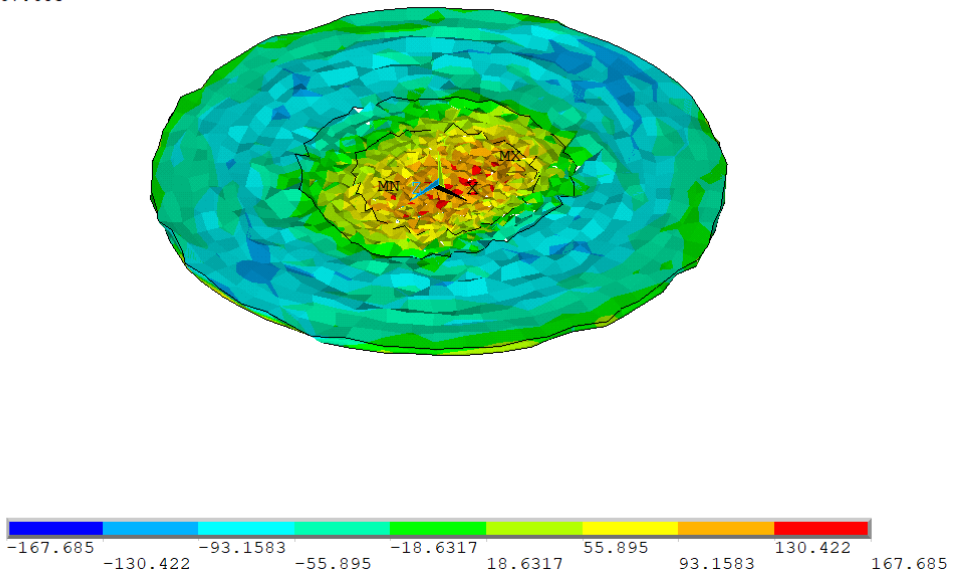

(a)
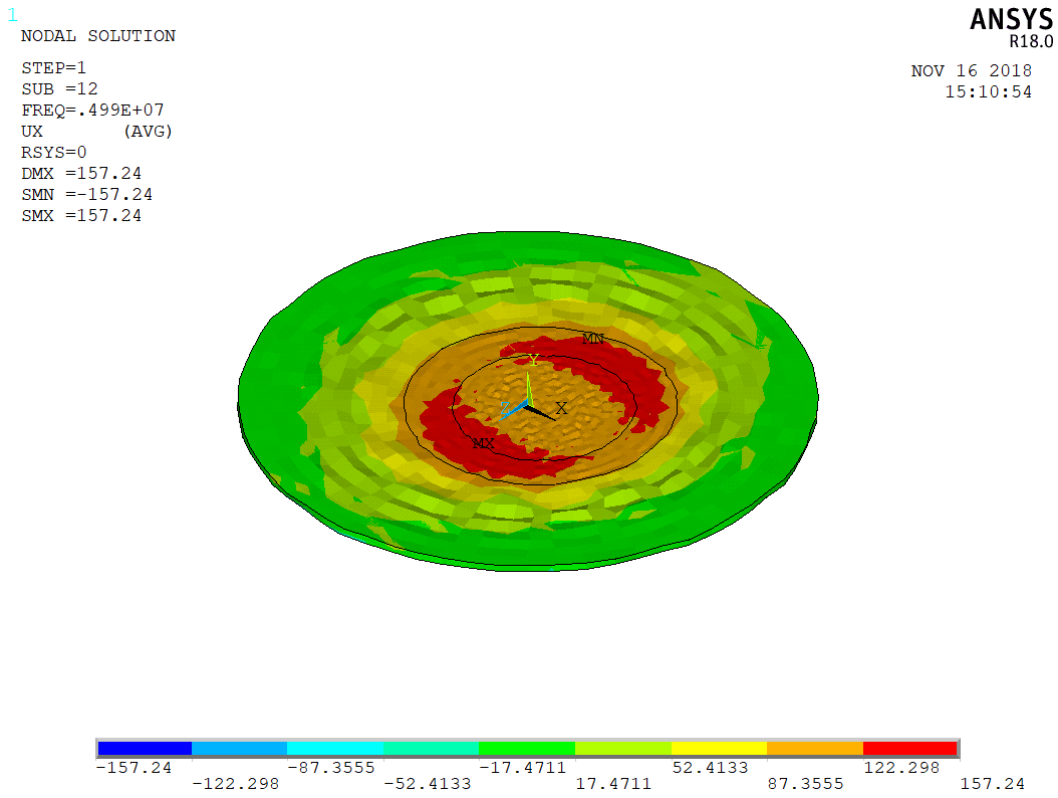

ov 162018

(b)

Figure 2. (a) The energy-trapping diagram of the 43rd modal subsets; (b) the energy-trapping diagram of the 12 th modal subset. 


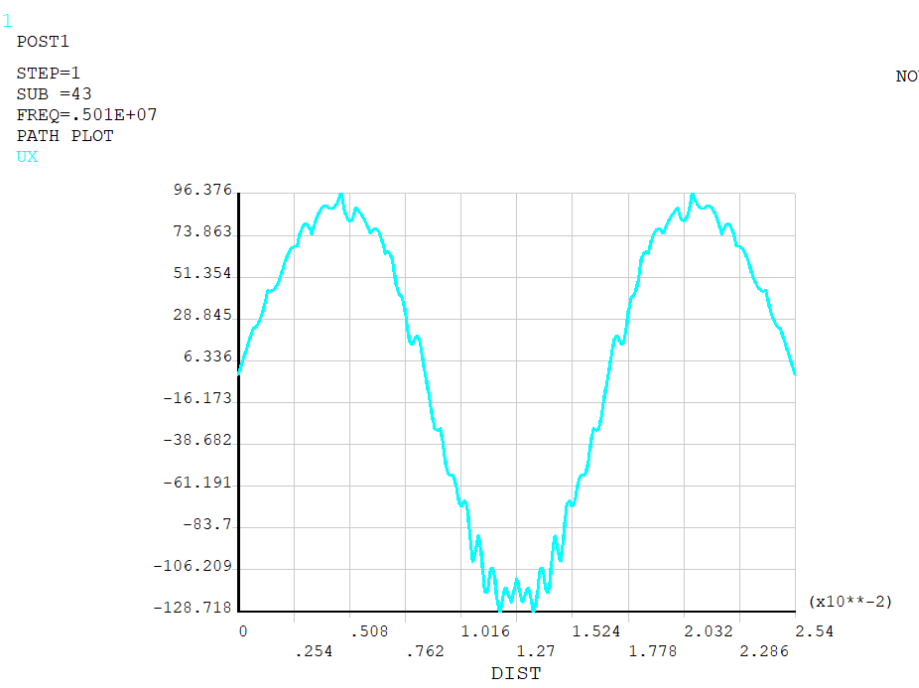

(a)

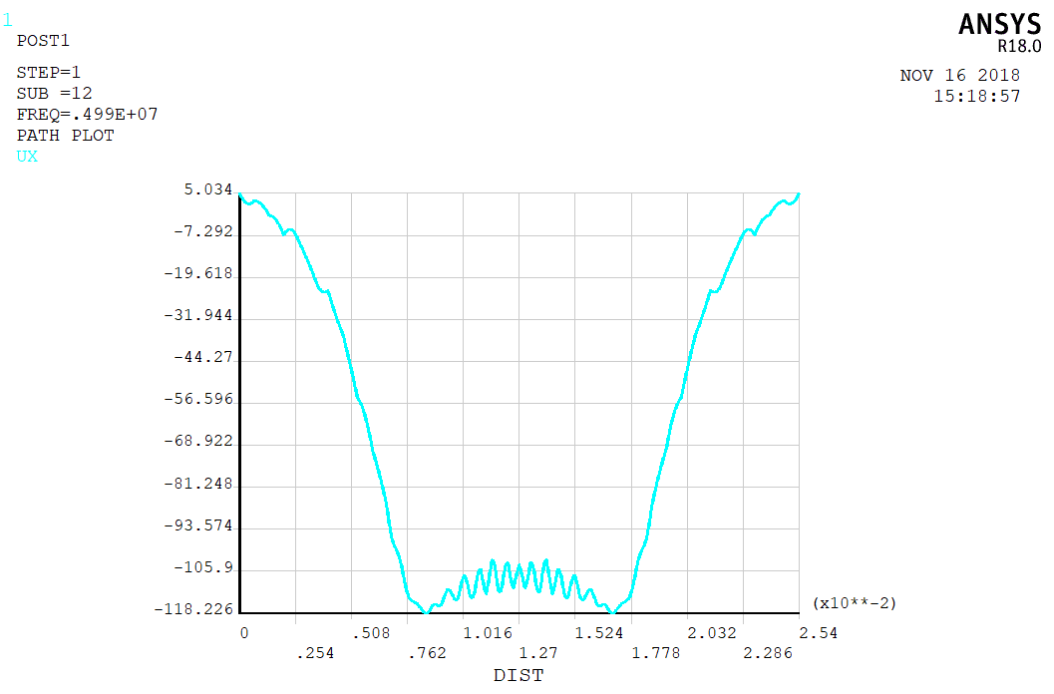

(b)

Figure 3. (a) The vibration displacement curve of the chip along the radius corresponds to Figure 2a; (b) the vibration displacement curve of the chip along the radius corresponds to Figure $2 \mathrm{~b}$.

In Figure 2a, the energy of harmonics transmits to the non-electrode region, and the trapping effect is not obvious in Figure 2a. Figure $2 b$ is more consistent with the best trap effect, and the maximum energy is confined to the central area of the electrode. Thus, the chip resonance is at $4.989680 \mathrm{MHz}$ with single oscillation frequency. Figure 3a shows other vibrational modes of the quartz crystal. Since this paper adopts a thickness shear mode, these vibrational modes do not meet the requirements. In Figure 3b, the vibrational displacement curve is generally a normal distribution curve. The displacement amplitude in the central region is relatively small, and the displacement amplitude of the electrode region is larger than that of the central region, but the difference between the two regions is not obvious, so the electrode size needs optimization.

We kept the ring-shaped gold electrode the same size, but the outer radius was increased to 8 and $10 \mathrm{~mm}$. The energy-trap diagram is shown in Figure 4, and Figure 5 shows the corresponding vibration displacement curve. The electrode frequencies are $4,983,940 \mathrm{~Hz}$ and $4,998,130 \mathrm{~Hz}$, respectively. We find that the optimal energy-trap graph is obtained when the outer diameter of the electrode is 
$8 \mathrm{~mm}$. Figure 5a shows the smoothest vibration displacement curve when the outer diameter is $8 \mathrm{~mm}$. Figure $5 b$ shows that the interference of the non-resonant frequency signal is more obvious in the central region, which has great influence on the oscillation frequency. Hence, the optimal size for the ring-shaped gold electrode is with $4 \mathrm{~mm}$ inner diameter and an $8 \mathrm{~mm}$ outer diameter. We find that the larger the outer diameter of the electrode ring, the greater the frequency of the electrode. The optimal size of the bottom electrode is $6 \mathrm{~mm}$, and we get the best energy-trapping diagram and corresponding vibration displacement curve in Figure 6.

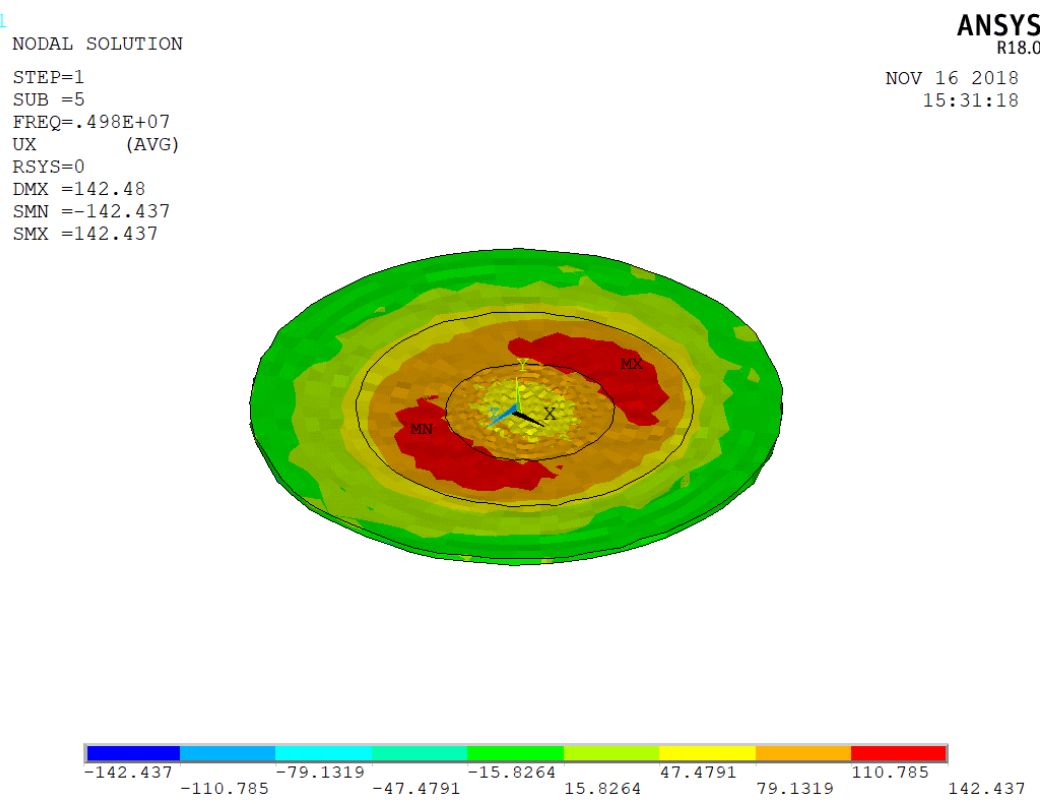

(a)
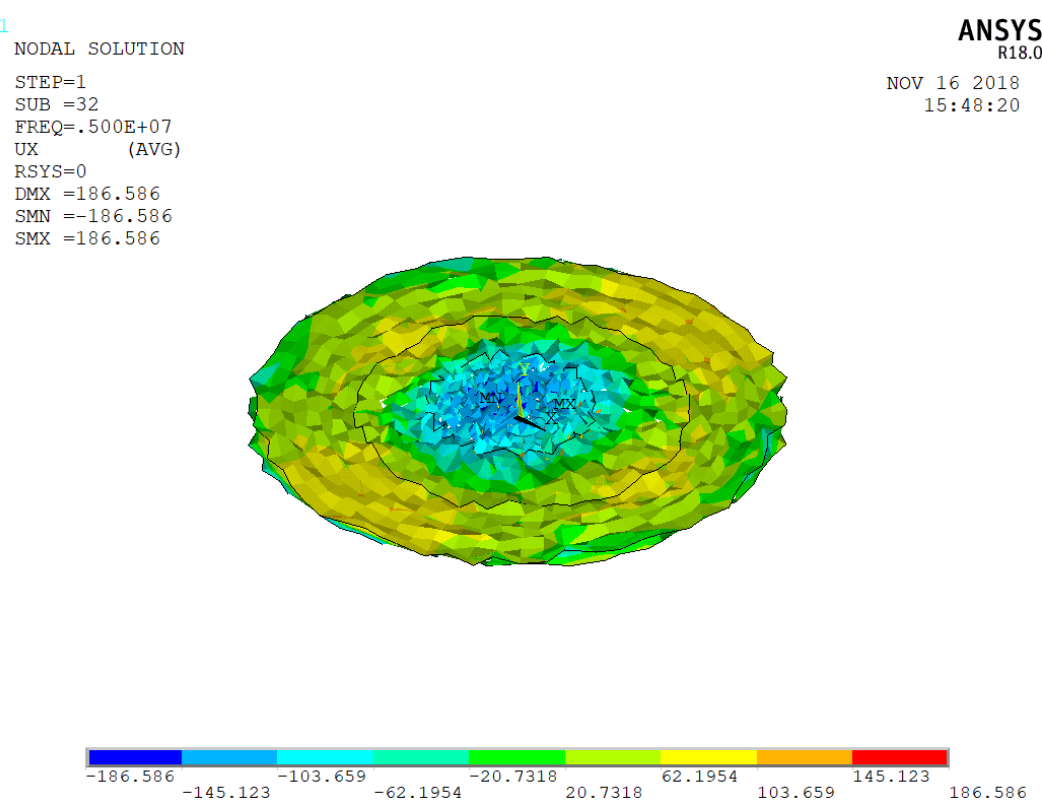

(b)

Figure 4. (a) The energy-trap graph corresponding to the external radius of $8 \mathrm{~mm}$; (b) the energy-trap graph corresponding to the external radius of $10 \mathrm{~mm}$. 


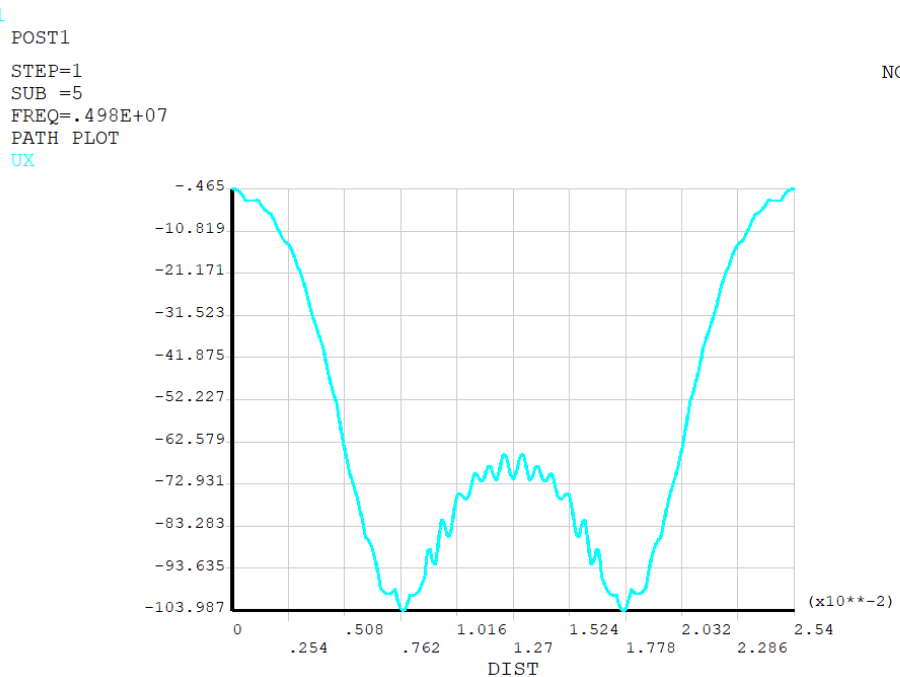

ANSYS

NOV $16 \quad 2018$

(a)

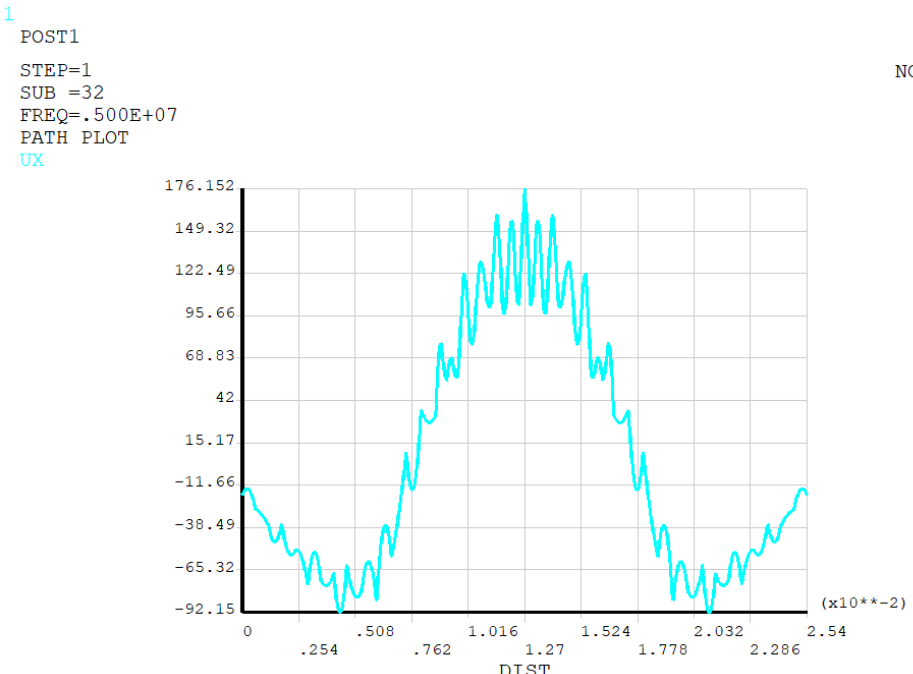

(b)

Figure 5. (a) The curve of vibration displacement at the outer diameter of $8 \mathrm{~mm}$; (b) the curve of vibration displacement at the outer diameter of $10 \mathrm{~mm}$. 

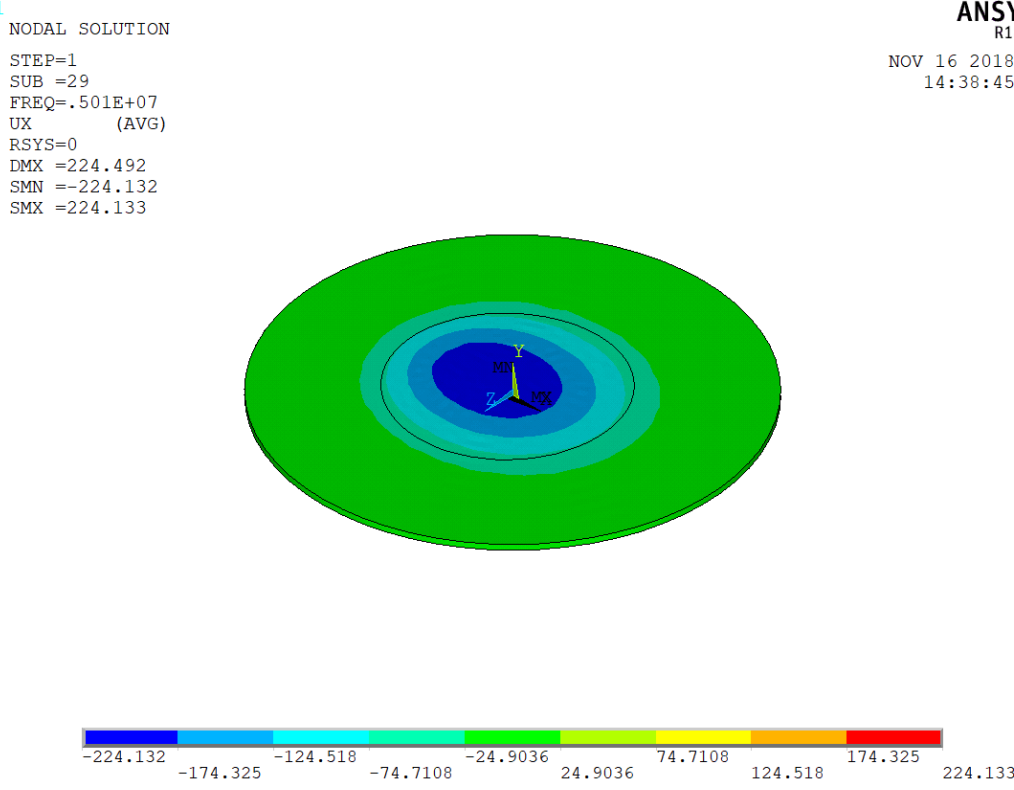

(a)

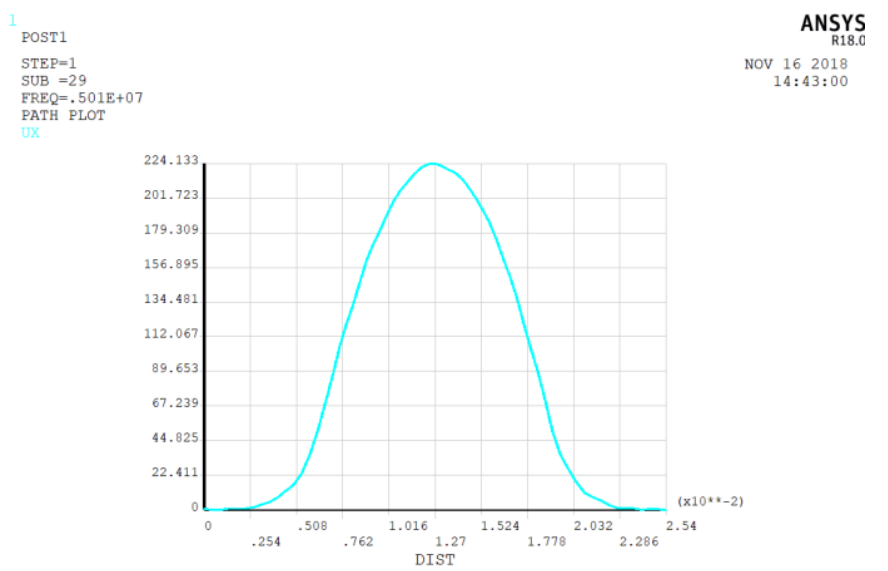

(b)

Figure 6. (a) The energy-trapping diagram of a circular gold electrode with a radius of $6 \mathrm{~mm}$; (b) the vibration displacement curve corresponding to a $6 \mathrm{~mm}$ circular gold electrode.

Combined with the influence of electrode size on the resonant frequency and vibration mode, the optimal size of the quartz crystal with the upper electrode as the ring shape and the lower electrode as the circular shape is $4 \mathrm{~mm}$ for the inner ring, $8 \mathrm{~mm}$ for the outer ring of the upper electrode, and $6 \mathrm{~mm}$ for the lower electrode.

\subsection{Spherical Nanoparticles}

We used the DDSCAT method [39-41] to study the absorption peaks and corresponding wavelengths of gold nanoparticles with different shapes and sizes in different media refractive indices. We control the same medium, which is benzene, under visible light of $530-550 \mathrm{~nm}$. The absorption spectra of spherical gold nanoparticles of different sizes at the incident light wavelength of 530-550 nm are obtained, as shown in Figure 7. 


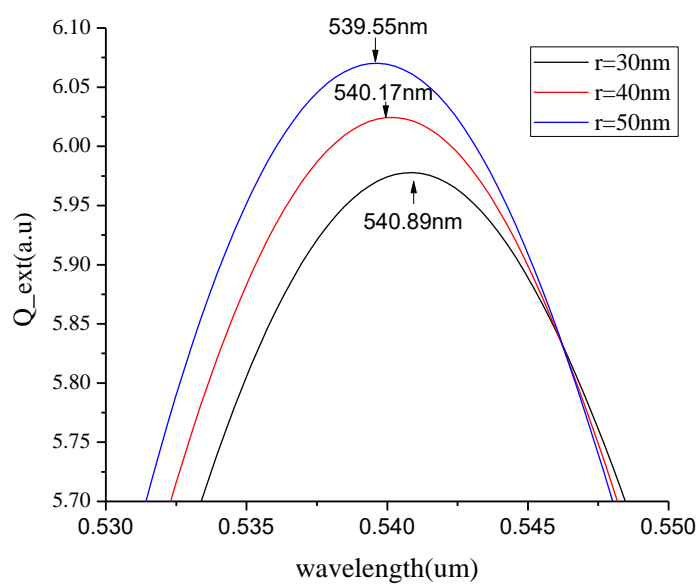

Figure 7. The absorption spectra of spherical gold nanoparticles of different sizes (30, 40, and $50 \mathrm{~nm})$ with incident light wavelength of 530-550 $\mathrm{nm}$.

It was obvious that with the increased radius of the spherical gold nanoparticles, the wavelength of the maximum absorption peak decreases. Moreover, obvious red shift occurs, and the maximum extinction efficiency was increasing. The absorption peak of $30 \mathrm{~nm}$ gold nanoparticles was 5.98 a.u., and the wavelength of the peak was $539.55 \mathrm{~nm}$. Finally, we compare the absorption spectra of ring nanoparticles with an inner radius of $20 \mathrm{~nm}$ and an outer radius of $30 \mathrm{~nm}$, and spherical nanoparticles with a radius of $30 \mathrm{~nm}$ in ethanol at an incident light wavelength of 510 to $550 \mathrm{~nm}$, and the results are shown in Figure 8.

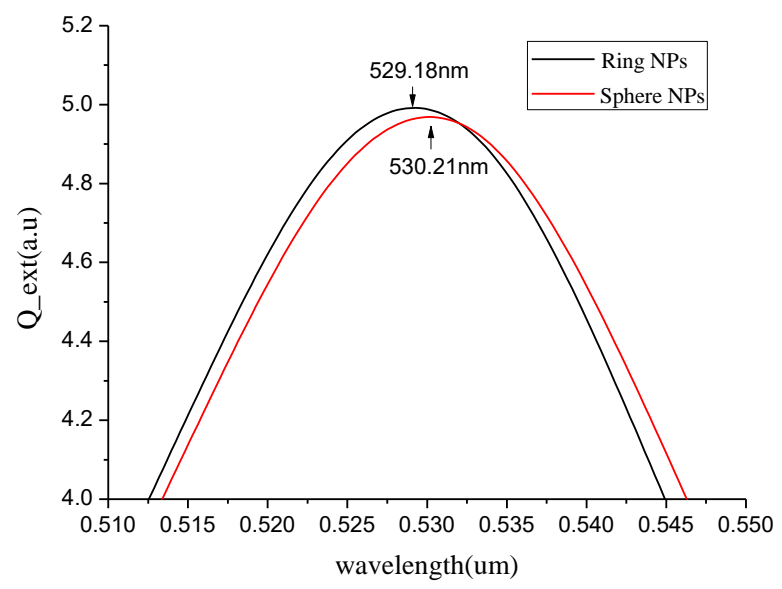

Figure 8. The absorption spectrum of ring nanoparticles and spherical nanoparticles at the wavelength of $510-550 \mathrm{~nm}$.

As shown in Figure 8, we know that the maximum extinction efficiency of the spherical nanoparticles is 4.992 a.u., and the corresponding wavelength is $529.18 \mathrm{~nm}$. The maximum extinction efficiency of the ring nanoparticles is 4.968 a.u., and the corresponding wavelength is $530.21 \mathrm{~nm}$. In the same volume, the ring nanoparticles were more efficient than spherical nanoparticles, but the corresponding wavelengths were smaller than spherical ones. In the case where the spherical radius and the outer annular radius were the same, the refractive sensitivity of the spherical nanoparticles is $61.34 \mathrm{~nm} / \mathrm{RIU}$, which is larger than the refractive sensitivity of the annular nanoparticles of $42.55 \mathrm{~nm} /$ RIU. Therefore, the LSPR sensor chip was designed by selecting spherical gold nanoparticles with a radius of $30 \mathrm{~nm}$ and a refractive index of $61.34 \mathrm{~nm} / \mathrm{RIU}$. 


\subsection{Effect of Spherical Nanoparticles on the Resonant Base Frequency of the Quartz Crystal ofGold Electrode}

We add the spherical gold nanoparticle load to the gold electrode and obtain the energy-trap diagram and corresponding vibration displacement curve of the quartz crystal model loaded with mass load using ANSYS software. We obtain the fundamental frequency variation of gold electrode quartz crystal, $\Delta f=8574.3 \mathrm{~Hz}$, and find that compared with the fundamental frequency, $f s=4.98 \mathrm{MHz}$, it is completely negligible, so the spherical gold nanoparticles have no effect on the resonant fundamental frequency of the quartz crystal chip.

\section{Results}

Frequency shift and optical spectrum of dual-technology sensor chip in air and three different liquids were calculated. The related properties of three different liquids (water, ethyl alcohol, and benzene) are seen in Table 1. We controlled for the same shape and size of particles (selecting $30 \mathrm{~nm}$ gold nanosphere particles) and obtained the absorption spectra in the refractive index of different media.

As Figure 9a shows, the maximum extinction efficiency increased with an increase of the refractive index of the liquid. The corresponding wavelength of absorption peak also increased with the increase of the refractive index of the liquid. The refractive index sensitivity was calculated by linear fitting in Figure 9b. The transverse axis is the refractive index of the four media, and the vertical axis is the corresponding peak position of each refractive index. The sensitivity of the refractive index is $61.34 \mathrm{~nm} / \mathrm{RIU}$. The refractive index of the media is 1, 1.33, 1.36, and 1.51 under visible light of $450-650 \mathrm{~nm}$, and the results are shown in Figure 9b. The resonant frequency has obvious changes when the sensor is in different liquids. The resonant frequency has a shift from 4,983,940 to $4,964,690 \mathrm{~Hz}$ when $\varepsilon_{r}$ varies from 2.27 to 78.30 .

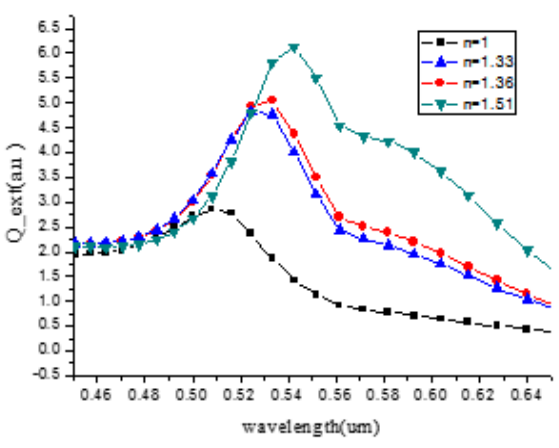

(a)

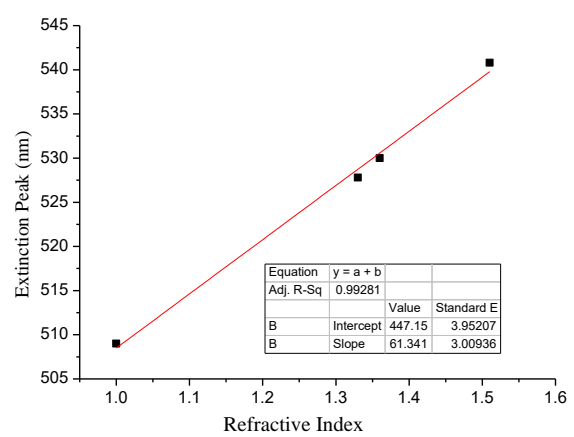

(b)

Figure 9. (a) The absorption spectra of the incident light at $450-650 \mathrm{~nm}$ and a radius of $30 \mathrm{~nm}$ in different media $(1,1.33,1.36$, and 1.51); (b) calculation of refractive index sensitivity for $30 \mathrm{~nm}$ nanoparticle array.

Table 1. QCM resonant frequency and frequency shift in different liquids.

\begin{tabular}{cccccc}
\hline Surrounding Medium & $\boldsymbol{n}$ & $\boldsymbol{\rho} \mathbf{( \mathbf { k g } / \mathbf { m } ^ { \mathbf { 3 } } )}$ & $\varepsilon_{\boldsymbol{r}}$ & $\boldsymbol{f} \mathbf{( H z )}$ & $\boldsymbol{\Delta} \boldsymbol{f} \mathbf{( H z )}$ \\
\hline Air & 1 & 1.29 & 1.00 & 4983940 & 0 \\
Water & 1.33 & 1000 & 78.30 & 4981470 & 2470 \\
Ethyl alcohol & 1.36 & 789 & 6.08 & 4977730 & 6210 \\
Benzene & 1.51 & 1880 & 2.27 & 4964690 & 19250
\end{tabular}

Liquid property: $n$ is the refractive index sensitivity of the liquid. $\rho$ is the density. $\varepsilon_{r}$ is the relative dielectric constant. $f$ is resonant frequency of QCM in different medium. $\Delta f$ is the frequency shift in different liquid compared with those in air.

\section{Conclusions}

Combining the above experimental results, we chose the ring gold electrode with an inner radius of $4 \mathrm{~mm}$ and an outer radius of $8 \mathrm{~mm}$ as the top electrode of quartz crystal, and a circular gold electrode 
with a radius of $6 \mathrm{~mm}$ as the bottom electrode. The vibration frequency of the chip is $4,983,940 \mathrm{~Hz}$. The vibrational displacement curve of the circular gold electrode is a normal distribution chart, while the central region of the vibration displacement curve of the annular gold electrode is concave, which is obviously different from the electrode region. We selected the spherical gold nanoparticles with a radius of $30 \mathrm{~nm}$ and a refractive sensitivity of $61.34 \mathrm{~nm} / \mathrm{RIU}$ to design the LSPR sensor chip. It is found, through experiments, that when the spherical gold nanoparticles are prepared in the middle of the ring-shaped gold electrode, there is no effect on the resonant base frequency of QCM. The purpose of the ring electrode is to make the central area transmit the light, which can be integrated in the structure of QCM and LSPR technology, forming dual-technology chips with ring electrode. The technique has good prospects for the molecular characterization of complex structures and high sensitivity for DNA and RNA detection.

Author Contributions: C.M. and J.Z. conceptualized, designed, and performed analog simulation. C.M. analyzed the data. C.M. and J.Z. researched resonant fundamental frequency. C.M. and J.Z. simulated nanoparticle spectra. X.L. and W.Z. designed detection system. C.M. and J.Z. together wrote the paper. All authors discussed the results and commented on the manuscript.

Acknowledgments: Authors thank the project of Natural Science Research of the colleges and universities in the Jiangsu Province of China (No. 17KJB510013, No. 15KJB310003), the National Natural Science Foundation of China (Grant No.61804068, Grant No.61671221, Grant No.61601206), the project of Zhenjiang Laboratory of Information Sensing and Transmission Technology for Smart Ocean (GX2017004) for sponsoring this paper.

Conflicts of Interest: The authors declare no conflict of interest.

\section{References}

1. Huang, X.; Bai, Q.; Hu, J.; Hou, D. A practical model of quartz crystal microbalance in actual applications. Sensors 2017, 17, 1785. [CrossRef] [PubMed]

2. Mohammadnejad, S.; Noaparast, M.; Hosseini, S.; Aghazadeh, S.; Mousavinezhad, S.; Hosseini, F. Physical Methods and Flotation Practice in the Beneficiation of a Low Grade Tungsten-Bearing Scheelite Ore. Russ. J. Non-Ferrous Met. 2018, 59, 6-15. [CrossRef]

3. Alassi, A.; Benammar, M.; Brett, D. Quartz Crystal Microbalance Electronic Interfacing Systems: A. Review. Sensors 2017, 17, 2799. [CrossRef] [PubMed]

4. Lei, O.; Tian, W.; Ma, B. High-sensitivity QCM with multielectrode piezoelectric quartz crystal design. Int. J. Appl. Ceram. Technol. 2018, 15, 751-757. [CrossRef]

5. Nomura, T.; Okuhara, M. Frequency shifts of piezoelectric quartz crystals immersed in organic liquids. Anal. Chim. Acta 1982, 142, 281-284. [CrossRef]

6. Kananizadeh, N.; Rice, C.; Lee, J.; Rodenhausen, K.B.; Sekora, D.; Schubert, M.; Schubert, E.; Bartelt-Hunt, S.; $\mathrm{Li}$, Y. Combined quartz crystal microbalance with dissipation (QCM-D) and generalized ellipsometry (GE) to characterize the deposition of titanium dioxide nanoparticles on model rough surfaces. J. Hazard. Mater. 2017, 322, 118-128. [CrossRef] [PubMed]

7. Kojima, T. Surface Modification Enhanced Reflection Intensity of Quartz Crystal Microbalance Sensors upon Molecular Adsorption. Anal. Sci. 2018, 34, 363-368. [CrossRef] [PubMed]

8. Huang, G.S.; Wang, M.T.; Hong, M.Y. A versatile QCM matrix system for online and high-throughput bio-sensing. Analyst 2006, 131, 382-387. [CrossRef] [PubMed]

9. Etchenique, R.; Buhse, T. Anomalous behaviour of the quartz crystal microbalance in the presence of electrolytes. Analyst 2000, 125, 785-787. [CrossRef]

10. Edvardsson, M.; Svedhem, S.; Wang, G.; Richter, R.; Rodahl, M.; Kasemo, B. QCM-D and reflectometry instrument: Applications to supported lipid structures and their biomolecular interactions. Anal. Chem. 2008, 81, 349-361. [CrossRef] [PubMed]

11. Cao, Y.; Griffith, B.; Bhomkar, P.; Wishart, D.S.; McDermott, M.T. Functionalized gold nanoparticle-enhanced competitive assay for sensitive small-molecule metabolite detection using surface plasmon resonance. Analyst 2018, 143, 289-296. [CrossRef] [PubMed]

12. Tang, D.; Li, Q.; Tang, J.; Su, B.; Chen, G. An enzyme-free quartz crystal microbalance biosensor for sensitive glucose detection in biological fluids based on glucose/dextran displacement approach. Anal. Chim. Acta 2011, 686, 144-149. [CrossRef] [PubMed] 
13. Lin, T.Y.; Hu, C.H.; Chou, T.C. Determination of albumin concentration by MIP-QCM sensor. Biosens. Bioelectron. 2004, 20, 75-81. [CrossRef] [PubMed]

14. Uludağ, Y.; Tothill, I.E. Development of a sensitive detection method of cancer biomarkers in human serum (75\%) using a quartz crystal microbalance sensor and nanoparticles amplification system. Talanta 2010, 82, 277-282. [CrossRef] [PubMed]

15. Chen, J.C.; Sadhasivam, S.; Lin, F.H. Label free gravimetric detection of epidermal growth factor receptor by antibody immobilization on quartz crystal microbalance. Process Biochem. 2011, 46, 543-550. [CrossRef]

16. Vashist, S.K.; Vashist, P. Recent advances in quartz crystal microbalance-based sensors. J. Sens. 2011. [CrossRef]

17. Sepúlveda, B.; Angelomé, P.C.; Lechuga, L.M.; Liz-Marzán, L.M. LSPR-based nanobiosensors. Nano Today 2009, 4, 244-251. [CrossRef]

18. Becker, B.; Cooper, M.A. A survey of the 2006-2009 quartz crystal microbalance biosensor literature. J. Mol. Recognit. 2011, 24, 754-787. [CrossRef] [PubMed]

19. Hao, D.; Hu, C.; Grant, J.; Glidle, A.; Cumming, D.R. Hybrid localized surface plasmon resonance and quartz crystal microbalance sensor for label free biosensing. Biosens. Bioelectron. 2018, 100, 23-27. [CrossRef] [PubMed]

20. Ferhan, A.R.; Jackman, J.A.; Cho, N.J. Integration of quartz crystal microbalance-dissipation and reflection-mode localized surface plasmon resonance sensors for biomacromolecular interaction analysis. Anal. Chem. 2016, 88, 12524-12531. [CrossRef] [PubMed]

21. Ebarvia, B.S.; Ubando, I.E.; Sevilla III, F.B. Biomimetic piezoelectric quartz crystal sensor with chloramphenicol-imprinted polymer sensing layer. Talanta 2015, 144, 1260-1265. [CrossRef] [PubMed]

22. Sauerbrey, G. Verwendung von Schwingquarzen zur Wägung dünner Schichten und zur Mikrowägung. Zeitschrift für physik. Zeitschrift für physik 1959, 155, 206-222. [CrossRef]

23. Klosterman, L.; Riley, J.K.; Bettinger, C.J. Control of Heterogeneous Nucleation and Growth Kinetics of Dopamine-Melanin by Altering Substrate Chemistry. Langmuir 2015, 31, 3451-3458. [CrossRef] [PubMed]

24. Yavas, O.; Svedendahl, M.; Dobosz, P.; Sanz, V.; Quidant, R. On-a-chip biosensing based on all-dielectric nanoresonators. Nano Lett. 2017, 17, 4421-4426. [CrossRef] [PubMed]

25. Agrawal, A.; Kriegel, I.; Milliron, D.J. Shape-dependent field enhancement and plasmon resonance of oxide nanocrystals. J. Phys. Chem. C 2015, 119, 6227-6238. [CrossRef]

26. Yan, Q.Q.; Qin, W.J.; Wang, C.; Song, P.F.; Ding, G.; Yang, L.Y.; Yin, S.G. Plasmon-enhanced polymer bulk heterojunction solar cells with solution-processable Ag nanoparticles. Optoelectron. Lett. 2011, 7, 410-414. [CrossRef]

27. Kelly, K.L.; Coronado, E.; Zhao, L.L.; Schatz, G.C. The optical properties of metal nanoparticles: The influence of size, shape, and dielectric environment. J. Phys. Chem. B 2003, 107, 668-677. [CrossRef]

28. Zhu, J.; Li, W.; Zhu, M.; Zhang, W.; Niu, W.; Liu, G. Influence of the pH value of a colloidal gold solution on the absorption spectra of an LSPR-assisted sensor. AIP Adv. 2014, 4, 031338. [CrossRef]

29. Wang, T.; Zhang, J.; Xue, P.; Chen, H.; Ye, S.; Wang, S.; Yang, B. Nanotransfer printing of gold disk, ring and crescent arrays and their IR range optical properties. J. Mater. Chem. C 2014, 2, 2333-2340. [CrossRef]

30. Luo, Y.; Xu, J.; Li, Y.; Gao, H.; Guo, J.; Shen, F.; Sun, C. A novel colorimetric aptasensor using cysteamine-stabilized gold nanoparticles as probe for rapid and specific detection of tetracycline in raw milk. Food Control 2015, 54, 7-15. [CrossRef]

31. Oćwieja, M.; Maciejewska-Prończuk, J.; Adamczyk, Z.; Roman, M. Formation of positively charged gold nanoparticle monolayers on silica sensors. J. Colloid Interface Sci. 2017, 501, 192-201. [CrossRef] [PubMed]

32. Maciejewska-Prończuk, J.; Oćwieja, M.; Adamczyk, Z.; Pomorska, A. Formation of gold nanoparticle bilayers on gold sensors. Colloids Surf. A Physicochem. Eng. Asp. 2019, 560, 393-401. [CrossRef]

33. Zhu, J.; Li, X.; Zheng, W.; Wang, B.; Tian, Y. Improved localized surface plasmon resonance index sensitivity based on chemically-synthesized gold nanoparticles on Indium Tin Oxide surfaces. Nanotechnology 2018, 29, 055701. [CrossRef] [PubMed]

34. Larsson, E.M.; Alegret, J.; Käll, M.; Sutherland, D.S. Sensing characteristics of NIR localized surface plasmon resonances in gold nanorings for application as ultrasensitive biosensors. Nano Lett. 2007, 7, 1256-1263. [CrossRef] [PubMed]

35. Tsai, C.Y.; Lu, S.P.; Lin, J.W.; Lee, P.T. High sensitivity plasmonic index sensor using slablike gold nanoring arrays. Appl. Phys. Lett. 2011, 98, 153108. [CrossRef] [PubMed] 
36. Kumar, S.; Yoon, S.H.; Kim, G.H. Bridging the nanogap electrodes with gold nanoparticles using dielectrophoresis technique. Curr. Appl. Phys. 2009, 9, 101-103. [CrossRef]

37. Choi, H.M.; Chang, J.Y.; Trinh, L.A.; Padilla, J.E.; Fraser, S.E.; Pierce, N.A. Programmable in situ amplification for multiplexed imaging of mRNA expression. Nat. Biotechnol. 2010, 28, 1208. [CrossRef] [PubMed]

38. Zhu, J.; Huang, S.; Ye, J.Y.; Zhang, X.Q.; Liu, G.H. Design of a quartz crystal with transparent electrode used for both QCM-D and LSPR technology. Sens. Actuators A Phys. 2015, 229, 141-146. [CrossRef]

39. Mu, H.; Lv, J.; Liu, C.; Sun, T.; Chu, P.K.; Zhang, J. Localized surface plasmon resonance properties of Ag nanorod arrays on graphene-coated Au substrate. Opt. Commun. 2017, 402, 216-220. [CrossRef]

40. Lee, H.; Ro, G.; Kim, J.M.; Kim, Y. Discrete-dipole approximation for the optical properties with morphological changes of silver nanoprism and nanosphere via galvanic reaction. Mater. Lett. 2017, 209, 138-141. [CrossRef]

41. Ren, Y.; Qi, H.; Chen, Q.; Wang, S.; Ruan, L. Localized surface plasmon resonance of nanotriangle dimers at different relative positions. J. Quant. Spectrosc. Radiat. Transf. 2017, 199, 45-51. [CrossRef]

(C) 2019 by the authors. Licensee MDPI, Basel, Switzerland. This article is an open access article distributed under the terms and conditions of the Creative Commons Attribution (CC BY) license (http:/ / creativecommons.org/licenses/by/4.0/). 